\title{
Fibonacci Harmonics: A New Mathematical Model of Synchronicity
}

\author{
Robert G. Sacco \\ Fibonacci Lifechart, Toronto, Canada \\ Email: drrobertsacco@gmail.com
}

How to cite this paper: Sacco, R.G. (2018) Fibonacci Harmonics: A New Mathematical Model of Synchronicity. Applied Mathematics, 9, 702-718.

https://doi.org/10.4236/am.2018.96048

Received: May 19, 2018

Accepted: June 26, 2018

Published: June 29, 2018

Copyright $\odot 2018$ by author and Scientific Research Publishing Inc. This work is licensed under the Creative Commons Attribution International License (CC BY 4.0).

http://creativecommons.org/licenses/by/4.0/

\begin{abstract}
This article aims to provide a brief overview of the relevance of new findings about the Fibonacci Life Chart Method (FLCM) for understanding synchronicity. The FLCM is reviewed first, including an exposition of the golden section model, and elaboration of a new harmonic model. The two models are then compared to illuminate several strengths and weaknesses in connection with the following four major criteria regarding synchronicity: explanatory adequacy; predictability of future synchronicities; simplicity of the model; and generalizability to other branches of knowledge. The review indicates that both models appear capable of simulating nonlinear and fractal dynamics. Hybrid approaches that combine both models are feasible and necessary for projects that aim to experimentally address synchronicity.
\end{abstract}

\section{Keywords}

Synchronicity, Dynamical Systems, Brain Dynamics, Fibonacci Life Chart Method, Golden Section Model, Harmonic Model

\section{Introduction}

Synchronicity is among the most mysterious experiences, involving a noncausal connection between mind and matter. Stories involving synchronicity appear in movies, biographies, theatre, and literature. It has been a perennial challenge to explain. This article places synchronicity in the context of a mathematical system in which mind and matter play complementary roles in a reality that has a mathematical structure [1] [2].

In recent decades, two main theoretical approaches have dominated the field of synchronicity. One of these emphasizes quantum determinants, as shaped by mind-matter entanglement [3]. The other emphasizes psychological determinants, as shaped by biased cognition [4]. Both have proposed to explain the 
meaningful coincidence between a thought and related event in the world. The quantum approach stresses the entanglement induced nonlocal correlations of quantum physics, conceiving of mind-matter entanglement as the hypothetical origin of mind-matter correlations and synchronistic phenomena. The biased cognition approach, generally based on poor probabilistic reasoning, has emphasized chance occurrences and our need to make sense of ourselves and the world. Thus, the disciplines of physics and psychology have been most prominent in guiding how researchers think about synchronicity.

This article turns to a different discipline, mathematics, to explain a theory of synchronicity. A mathematical approach to synchronicity was defined by Sacco [5] based on the Fibonacci Life Chart Method (FLCM). The FLCM has four main assumptions. First, human development is best framed within a nonlinear dynamical systems paradigm with the Fibonacci sequence driving changes in developmental timing relative to fixed points in the life cycle. Second, such patterns describe an eight-stage development process. Third, the transition from one stage to another is characterized by nonlinear dynamics (e.g., self-organization, emergence, attractors, fractals, complexity, and chaos). Fourth, during transitions, people seek new information and explore more adaptive configurations until they settle into a new stable state (attractor). In adopting such an approach, the theory is mathematical in the sense that it looks at principles of pattern formation and change across scientific disciplines and systems as diverse as cells, neurons, and the economy.

Although applying mathematical principles to synchronicity may seem novel, the notion that synchronicity might depend on the Fibonacci numbers was anticipated by Jung in a letter on February 9, 1956 [6]. Jung did not specify how Fibonacci numbers caused synchronicity, but he recognized the conceptual value of postulating that synchronicity operated based on the Fibonacci numbers because of their ubiquity in nature. Mathematical models are increasingly being invoked in psychology. For example, dynamical systems theory helps to analyze a broad range of cognitive and affective dynamics, interpersonal and group dynamics, and personality dynamics [7]. Likewise, fractal patterns are found across the domains of psychology including the brain, visual search, speech patterns, memory retrieval, interpersonal relationships, and personality [5]. Thus, the evidence is accumulating to suggest that mathematics furnishes a useful basis for making predictions about how people will think, feel, and act.

Previous attempts to apply dynamical systems theory to psychology have neglected one crucial aspect, which will be featured in this article: the points of system transition. At any given time, a dynamical system has a state given by a numerical phase space and a rule of evolution specifying trajectories in this space [8]. The evolution rule of the dynamical system describes what future states follow from the current state, and only when researchers identify this rule, can they study what is changing and the factors that catalyze this change. To make the theory predictive, mathematical terms must be defined according to an ordering 
scheme by which emergence and self-organization as principles of unique and spontaneous order can arise. Here the mathematical framework of Fibonacci numbers is used to describe precise points at which psychological experiences will shift.

There are two main parts to this article. The first will shed light on synchronicity by drawing on recent work on the FLCM. The golden section model of deriving time intervals is described, and an attempt will be made to develop and elaborate a new mathematical model of synchronicity from a harmonic perspective. The second section will then compare findings about the two models as a way of evaluating their capacity to explain and predict synchronicity phenomena.

\section{Description of the Fibonacci Life Chart Method}

Fibonacci Life Chart Method (FLCM) is a theoretical model of human development as a nonlinear dynamical system based on the Fibonacci sequence [5] [9]. Central to this conceptual framework is the notion that the Fibonacci sequence and ratio create fractal patterns in growing self-organizing systems. For example, this ratio (1.618) is found in phenomena ranging from the human body to snail shells to the spirals of the galaxies. The spiral cochlea of the ear is a Fibonacci spiral as is the spiral of the umbilical cord. Moreover, the nucleotide spirals of our DNA have Fibonacci proportions. The progression of the Fibonacci numbers and ratio are well suited to describing organic growth because they have the property of "gnomonic growth". Gnomonic growth implies that only the size changes, while the shape remains constant, as organisms maintain their overall shape and proportions as they grow. Thus, gnomonic growth relates to the aspect of self-similarity [5].

Crucially, FLCM assumes that each Fibonacci number represents a complete cycle of 24 hours. The value of FLCM depends on this basic assumption, and in part is based on the empirical observation that the developing human embryo doubles every 24 hours [10]. By applying this clock cycle to human development, one can make predictions about how human development will proceed. The timing of developmental events, for example, will depend on the self-organizing fractal properties of the Fibonacci sequence. Table 1 shows the FLCM generates a pattern characterized by eight age groups: Early Infancy ( 0 - 2 years), Toddler (2 - 4 years), Early Childhood (4 - 7 years), Middle Childhood (7 - 11 years), Adolescence (11 - 18 years), Young Adulthood (18 - 29 years), Middle Adulthood (29 - 48 years), and Older Adulthood (48 - 78+ years) [9]. These chronological ages, both higher and lower in frequency, are all golden ratio (1.618) to the next and previous in the sequence. The golden ratio "Phi" is also called the "scaling ratio".

Most notably, because the Fibonacci sequence relates to the well-known period doubling in dynamical systems theory [11], a bifurcation with a shift in coherence from one FLCM age to another occurs in concert with the onset of 
Table 1. The Fibonacci life chart method.

\begin{tabular}{|c|c|c|}
\hline Fn & Date & Chronological Age \\
\hline 0 & $1 / 01 / 00$ & 0.00 \\
\hline 1 & $1 / 02 / 00$ & 0.00 \\
\hline 1 & $1 / 03 / 00$ & 0.01 \\
\hline 2 & $1 / 05 / 00$ & 0.01 \\
\hline 3 & $1 / 08 / 00$ & 0.02 \\
\hline 5 & $1 / 13 / 00$ & 0.03 \\
\hline 8 & $1 / 21 / 00$ & 0.05 \\
\hline 13 & $2 / 03 / 00$ & 0.09 \\
\hline 21 & $2 / 24 / 00$ & 0.15 \\
\hline 34 & $3 / 29 / 00$ & 0.24 \\
\hline 55 & $5 / 23 / 00$ & 0.39 \\
\hline 89 & $8 / 20 / 00$ & 0.64 \\
\hline 144 & $1 / 11 / 01$ & 1.03 \\
\hline 233 & $9 / 01 / 01$ & 1.67 \\
\hline 377 & $9 / 13 / 02$ & 2.70 \\
\hline 610 & $5 / 15 / 04$ & 4.37 \\
\hline 987 & $1 / 27 / 07$ & 7.08 \\
\hline 1597 & $6 / 12 / 11$ & 11.45 \\
\hline 2584 & 7/09/18 & 18.53 \\
\hline 4181 & $12 / 19 / 29$ & 29.99 \\
\hline 6765 & $6 / 27 / 48$ & 48.52 \\
\hline 10946 & $6 / 16 / 78$ & 78.51 \\
\hline
\end{tabular}

deterministic chaos. The central point to the FLCM analysis of human development is that human development depends on the self-organizing properties of the Fibonacci sequence. When the Fibonacci numbers progressively increase, therefore, the self-organizing and fractal properties result in biological and psychological phase transition. Phase transitions can result from self-organization processes at multiple levels (e.g., molecules, genes, cell, organ, organ system, organism, behavior, and environment) that can influence each other. The self-organized hierarchy begins at the mathematical and quantum level. This suggests that the ultimate nature of reality is mathematical [1] [2]. If mathematics unifies mind and matter, then it is possible to model information transmission regardless of mental or physical properties.

Although the premise that Fibonacci numbers can explain synchronicity invokes a mathematical model to explain human experience and is therefore essentially a mathematical theory, ironically its most famous advocate came from Carl Jung, a Swiss psychiatrist. Jung observed that “...synchronicity... is a sec- 
ondary effect of the primary coincidence of mental and physical events (as in the Fibonacci series). The bridge seems to be formed by the numbers" (p. 288) [6]. By "bridge" he meant the capacity to link mind and matter, although to be sure he did not specify how Fibonacci numbers could bridge these two worlds. Indeed, he provided little elaboration for his theory that Fibonacci numbers explain synchronistic events, a deficiency that this article seeks to address (aided by the substantial amount of empirical data on Fibonacci numbers in the decades since Jung's work). Nonetheless, Jung put forth the observation that synchronicity is essentially something controlled by the Fibonacci numbers.

\subsection{Model 1: Golden Section Model}

The FLCM goes beyond the simple primary intervals produced by the Fibonacci sequence. Just as the ratios of adjacent Fibonacci numbers approximate the golden ratio, the golden ratio may be used to compare adjacent primary intervals, to produce various secondary and tertiary time intervals (for a detailed description of this model, see [5]). Euclid gave the first written description of the golden ratio in connection with dividing a line segment into two unequal parts, such that the whole is to the long part as the long is to the short. Dividing a line segment with these proportions is known as creating a "golden section" or "golden cut". Figure 1 shows the interior and exterior golden section procedure based on two primary intervals (January 27, 2007 and June 12, 2011, see Table 1). After dividing the segment, two new secondary intervals appear (Date $\mathrm{X}$ and Date $\mathrm{Y}$ ), left and right of the original two endpoints.

Insofar as this is a valid procedure, the golden section points (interior and exterior) of the two new endpoints (Date $\mathrm{X}$ and Date $\mathrm{Y}$ ) can be used to calculate new tertiary and higher intervals. Treating calendar dates as applicable to the golden section means that this proportion-based system will endow the age distribution with a self-organized fractal structure [5]. A fractal is a recurring similar pattern at different scales. Many objects in nature are best described as fractals. The fractal structure is ubiquitous not only in trees, rivers, mountains, islands, and coastlines but also in human-made artifacts such as cities, streets, buildings, social media, and the Internet. All these constitute fractals as a set or pattern with far more small things than large ones. Fractals are said to be "self-similar": any subsystem of a fractal system reflects the whole system. Fractals belong to a set of models in which the threshold for activity, rather than being a function of a purely local variable, depends on nonlocal properties of the self-organizing structure [12]. Furthermore, all fractal structure involves the

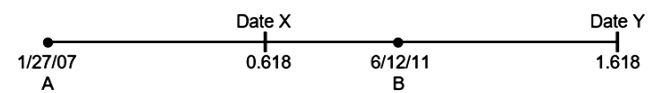

Figure 1. Golden section model of computing secondary intervals shows dividing primary intervals line segment $\mathrm{AB}$ by interior division according to the golden ratio $(0.618)$ to produce Date $\mathrm{X}$, also dividing primary intervals by exterior division according to the golden ratio (1.618) to produce Date Y. 
golden ratio because of its unique nesting capability, as seen for example in the Mandelbrot set [13].

Thus, the first prediction based on the golden section model (GSM) is that synchronistic events associated with nonlocality will be more likely at the golden section points. People will experience synchronicity near these points in time, but will not at other points in time (except perhaps in highly unusual circumstances). The bottom line is that golden section points have predictive value. Religious, spiritual, mystical, and synchronistic phenomena and similar experiences will have neural underpinnings triggered by the dynamical interaction between biology and mathematics [14]. Put another way, the formation and continuation of a series of synchronicities depends on whether their trajectories evolve by the rule of Fibonacci numbers than random chance; whether the synchronicity corresponds to nonlinear change than linear change, and so forth.

\subsection{Model 2: Harmonic Model}

The most fundamental characteristic of natural phenomena is the principle of cycles. A cycle is a regularly occurring sequence of events. Cycles exist in nature, economy, and biology. Cycles in nature include the day/night cycle, the four seasons, and solar activity. The basic business cycle encompasses an economic downturn, bottom, economic upturn, and top. Cycles are also part of the human body in the circadian rhythm, menstrual cycle, and brain waves. The harmonic model (HM) of synchronicity endows those cycles with much power and importance and is an alternative approach to computing secondary time intervals. One crucial feature of the HM is the time-periodic patterns of the primary intervals. The primary intervals are not divided into secondary (and higher) time intervals as in the golden section model. Rather, the FLCM primary intervals become part of a harmonic system, just as harmonics have a periodic series of cycles repeating in a sinusoidal fashion.

Harmonics relate to standing waves [15]. A standing wave pattern is not actually a wave, but rather a pattern of a wave. Thus, it does not consist of crests and troughs, but rather nodes and antinodes. The pattern is the result of the perfectly timed interference of two waves (sometimes more) of the same frequency with different directions of travel. The waves interfere in a way that produces points of no displacement at constant intervals. These points have the appearance of standing still and are referred to as nodes. In a standing wave, the nodes are a series of locations at equally spaced intervals where the wave amplitude (motion) is zero. At these points, the two waves add with opposite phase and cancel each other out. This condition is known as resonance. Standing waves are always associated with resonance. At frequencies other than a harmonic frequency, the interference of reflected and incident waves leads to a disturbance that is irregular, non-repeating, and non-resonant.

To the extent that human life cycles are a Fibonacci resonance phenomenon, time-periodic patterns can be described by phase relationships (phase patterns) 
among the primary intervals of the FLCM. Table 2 summarizes the features of the standing wave patterns for the nine key harmonics of the human lifespan. In this pattern, the first harmonic or fundamental wavelength is age 78.51 based on average life expectancy at birth of 78.6 years [16]. The fundamental wavelength has only one node from the birth date. The number of each successive harmonic expresses its wavelength as a power of Phi fraction of the fundamental wavelength: Harmonic $3=78.51 / 29.99$ (i.e., 2.62) of the fundamental wavelength. Based on a life expectancy of 78.6 years, the harmonic series consists of 240 unique nodal and antinodal points (262 including the birthdate and primary intervals). Note that each consecutive harmonic has a close resonance to the powers of Phi. The powers of Phi have unusual properties in that they relate not only exponentially, but are additive as well (e.g., $\mathrm{Phi}^{3}=\mathrm{Phi}^{2}+\mathrm{Phi}^{1}$ ).

Standing wave harmonics can occur in one, two, and three dimensions. An example of a harmonic wave in one-dimension is a guitar string. The wavelength of the standing wave for any given harmonic relates to the length of the string (and vice versa). These frequencies are related by whole-number divisions of its length $(1 / 2,1 / 3,1 / 4,1 / 5,1 / 6$, etc.) producing a series of harmonics whose frequencies are inversely proportional $(2 x, 3 x, 4 x, 5 x, 6 x$, etc., where $x$ is the fundamental frequency of the string) to those fractional divisions. Unlike standing waves in one-dimensional media such as waves in a string or sound waves in a pipe, the resonance frequencies of standing waves in two dimensions are not simple integer divisions (multiples) of the fundamental frequency. These harmonics are called "non-integer harmonics" or "interharmonics". Standing waves in two dimensions have been studied extensively as Chaldini patterns.

The most significant example of standing waves in three dimensions is the orbitals of an electron in an atom. On the atomic scale, it is more appropriate to describe the electron as a wave than as a particle. Electrons are bound to the space encompassing a nucleus similar to the way that a guitar string constrains the waves in the string. The constraint of a string in a guitar forces the string to vibrate with particular frequencies. In like manner, an electron can only vibrate

Table 2. Numerical patterns associated with standing waves.

\begin{tabular}{ccccc}
\hline Harmonic & \multicolumn{1}{c}{$\mathrm{Phi}^{\mathrm{n}}$} & \# of Nodes & \# of Antinodes & Interval (Years) \\
\hline 9th & $\mathrm{Phi}^{1}=1.6180$ & 47.01 & 47.00 & 1.67 \\
8th & $\mathrm{Phi}^{2}=2.6180$ & 29.08 & 29.00 & 2.70 \\
7th & $\mathrm{Phi}^{3}=4.2361$ & 17.97 & 18.00 & 4.37 \\
6th & $\mathrm{Phi}^{4}=6.8541$ & 11.09 & 11.00 & 7.08 \\
5th & $\mathrm{Phi}^{5}=11.0902$ & 6.86 & 7.00 & 11.45 \\
4th & $\mathrm{Phi}^{6}=17.9443$ & 4.24 & 4.00 & 18.53 \\
3rd & $\mathrm{Phi}^{7}=29.0345$ & 2.62 & 3.00 & 29.99 \\
2nd & $\mathrm{Phi}^{8}=46.9787$ & 1.62 & 2.00 & 48.52 \\
1st & $\mathrm{Phi}^{9}=76.0132$ & 1.00 & 1.00 & 78.51 \\
\hline
\end{tabular}


with particular frequencies. For an electron, these frequencies are determined by the fine-structure constant denoted by $\alpha$ (the Greek letter alpha). The value of $\alpha$ is approximately $1 / 137$. The fine-structure constant is an expression of Phi: $\mathrm{Phi}^{2} / 360=2.618 / 360=1 / 137.508=0.00727$ [17]. Indeed, the golden ratio exists at the quantum level in the magnetic resonance of atoms [18]. This suggests that the golden ratio essentially determines how an atom holds together its electrons.

At the deepest level, the human mind may manifest not only local but also nonlocal characteristics [3]. One source of support for this view was provided by the quantum holographic model of consciousness proposed by Edgar Mitchell [19]. Mitchell and Staretz [19] described holographic processing in terms of the brain's sensitivity to the phase of the electromagnetic waves of an object. They specifically drew on a resonance condition interpreted as a standing wave between the object and the brain. This resonance process is called phase conjugate adaptive resonance (PCAR). With a strong emotional response or focused attention in a person, some waves emitted from an object (i.e., subatomic particle, inanimate or animate thing, cells of human body) initiate into a nonlocal resonance process, whereas the absence of an emotional response or focused attention does not establish the resonance condition for decoding the object's nonlocal information structure. Extrasensory perception has a similar resonance effect, according to this model.

In short, we may regard Fibonacci harmonic intervals as standing waves in which the brain and quantum field exchange information across the nodes and antinodes of the interference. The human brain acts as the medium of information exchange with the phase of the receptors in the brain bringing about the resonance that enables the transmission of information from the quantum field to the brain. The quantum field acts as the source of nonlocal information stored in the nodes and antinodes of the interference patterns [19]. Both the brain and quantum field are in phase conjugate adaptive resonance, but whether this resonance involves local or nonlocal information depends on the emotional state of the person and Fibonacci harmonic intervals.

\section{Model Evaluation and Comparison}

Having described the two models, we can now evaluate and compare them. In each section following, the goal is to examine strengths and weaknesses of the models. The selected criteria for making comparisons between the different models follow general criteria published in the literature. Model evaluation criteria include: 1) explanatory adequacy (whether the theoretical account of the model helps make sense of observed data; 2) predictability (whether the model provides a good predictor of future observations); 3) simplicity (whether the model's description of observed data is the simplest possible), and 4) generalizability (whether the model provides a deeper insight or link to another branch of knowledge). To be preferred, the more criteria satisfied, the better. Although each criterion can be evaluated on its own, in practice, they are rarely indepen- 
dent of one another. Consideration of all four is necessary to assess the adequacy of a model.

\subsection{Explanation}

To explain a phenomenon, three issues need clarification [20]. First, it is important to know the causes of a particular phenomenon. Second, one would also need to determine whether the hypothesized causes correlate with the observed effect and whether that covariation is deterministic or probabilistic. Third, whether hypothesized causes precede the observed effects in time requires empirical assessment. Hence scientific models of synchronicity must be able to meet the demands of these three conditions.

Although few have discussed synchronicity as a scientific concept (For exceptions, see [21] [22] [23]), this view is explicit in Jung's writings. For example, Jung states that "Synchronicity is not a philosophical view but an empirical concept which postulates an intellectually necessary principle" (p. 96) [24]. At first, there seems to be a critical gap between the mental and the physical: How can these categories be compatible? The gap between the two categories is closed by bridge principles. Jung postulated the Fibonacci numbers as a bridge principle to explain synchronicity phenomena [6]. It is this bridge principle that creates the possibility of formulating quantitative predictions. Hence by taking the Fibonacci numbers as a bridge principle into account, both models (GSM and HM) provide explanatory power with experimentally testable formats in terms of Fibonacci time patterns.

However, there is also an important difference in the models regarding explanation of synchronicity. The GSM secondary (and higher) intervals are conjectured to correspond to synchronicity in terms of bifurcations that accompany the onset of chaos, where bifurcations are based on the golden ratio. The HM would, however, propose that the mechanism of synchronicity is via resonant self-organization, where nodes and antinodes represent periodic attractors based on Fibonacci harmonics. In the HM model, primary intervals form a harmonic system (Table 2) based on standing wave resonance patterns with no tertiary calculations necessary. The HM capitalizes on brain states as harmonic modes of the brain's structural connectivity and the synchronicity of cortical activity patterns into these harmonic brain states [25]. The set of all Fibonacci harmonics thus provides a new functional basis to explain synchronicity, which is based on the structural connectivity of the human brain. In fact, the brain's recurring electromagnetic activities form a highly reproducible harmonic function based on the golden ratio [26].

To infer a causal relationship between two variables or phenomena, both must be correlated. In other words, if A is correlated to $B$, then we should see a relationship between A and B. Covariation can either be deterministic or probabilistic. Deterministic covariation is when manipulation of only one variable produces an effect in another variable. Here, the cause is a necessary and sufficient 
condition for the effect. Probabilistic covariation is when an effect is a function of two or more causes. Here, none of the causes can be inferred from the other, and none of the causes is sufficient. Paradoxically, in dynamical systems theory, deterministic and probabilistic descriptions are often complementary [8]. Dynamical systems containing bifurcations can have both deterministic (universal and necessary) and probabilistic (historical happenstance) elements; they can possess varying degrees of susceptibility to change induced by fluctuations and they can respond differently to local, regional, and global fluctuations [8].

Finally, temporal ordering demands that to infer a causal relationship between two variables, the cause must always occur first before the effect. In other words, if $A$ causes $B$, then we should see a change in A first, and then a responding change to $\mathrm{B}$. As regards temporal ordering, it is more difficult to talk about the models and synchronicity interaction in the sense of causal relations. The notion of "probabilistic causality" should be preferred. Probabilistic causality attempts to explicate causal relationships in terms of probabilistic relationships [27]. This view is equipped to deal with the inherently stochastic properties associated with quantum mechanics. The central idea behind probabilistic causality is that causes change the probabilities of their effects. Therefore, no temporal ordering occurs essentially. However, there should be a "match" between Fibonacci time patterns and synchronicity to some extent. Several other variables (e.g., emotional intensity, focused attention) may raise the probabilities of a synchronistic event. Empirical studies are needed to verify the proposed relation between Fibonacci time patterns and synchronicity.

\subsection{Prediction}

Prediction deals with accurate anticipation of future occurrence of an event. By studying the trend of events regarding a phenomenon, researchers can forecast or predict what next will happen. The FLCM appears to be a reliable and valid predictor of biological and psychological change [9]. That is why the dynamical aspects of FLCM ages are also conjectured to relate to other discontinuous patterns, like synchronicity. A study by Sacco [14] explored the relationship between FLCM primary ages 11, 18, and 30 and religious and spiritual experience reports among young adults. Specifically, it was found that age 18 predicted a higher frequency of religious and spiritual experience. Further analysis of case reports at age 18 revealed that most involved heightened emotional experiences associated with crisis events, suggestive that people engage in meaning-making during times of instability, rather than times of stability [28].

Although both models use Fibonacci measurements, their age predictions are not identical. The GSM secondary (and higher) points are generated from the property that any interval between and outside adjacent points of the primary intervals must be at the golden ratio interval (0.618 and 1.618). In contrast, the $\mathrm{HM}$ describes secondary points as the nodes and antinodes of equally spaced primary intervals where the wave amplitude (motion) has zero displacement in a 
standing wave pattern. Thus, the GSM and HM differ only in secondary points. The theoretical exposition noted that when people are at primary and secondary golden interval points, there are discontinuous forces, such that people may feel a change in their thoughts, emotions, or behaviors, whereas at other points people maintain a high sense of stability because the dynamical phase space is continuous and they are outside the beginning and end points of discontinuities.

As the primary and secondary points exhibit a temporal structure with fractal and nonlinear features, a person may experience synchronicity as a discontinuity in the relationship between present conditions and future states [5]. Jung wrote that "Synchronicity is no more baffling or mysterious than the discontinuities of physics" (p. 102) [24]. In physics, a process becomes discontinuous in space and time at the very smallest of length or time scales. Discontinuities are, therefore, expected to involve events in space-time at the Planck length. The Planck length is the length scale at which the structure of space-time becomes dominated by quantum effects. The golden ratio, which to two decimal places is 1.62 , corresponds to the digits of the Planck length, $1.62 \times 10^{-33}$. Thus, on theoretical grounds, golden ratio time intervals could predict discontinuities in space and time, mind-matter correlations, and synchronistic phenomena. One problem to understand is the passage from a continuous to a discontinuous state. Is there an abrupt change or gradual transition?

Regarding the HM, a useful basis for making predictions about how people will think, feel, and act is the energy transfer and amplitude of the standing wave pattern. The total energy of a standing wave oscillates between a maximal value at zero displacement (node) and zero value at maximal displacement (antinode). This conclusion presents us with an immediate prediction: Energy transfer that changes the entire phase space may be amplified at nodes and antinodes, and the most important phase space changes may occur at these points. As described earlier, many factors may become intertwined in a resonance condition between the world and the brain, as the standing wave accumulates experiences, emotions, and commitments (some of which may be more immune to change). Of particular interest is that standing wave patterns can have varying amplitude: Amplitude measures how much energy is transported by the wave. Specifically, the amplitude of waves in the harmonic model increase according to powers of Phi. Put another way, as the amplitude of the waves get larger as the person ages there may be more energy associated with them.

\subsection{Simplicity}

To consider the GSM first, for simplicity, this model extends the original primary intervals (21 dates) to two iterations of measured points (112 dates). The model computes measured points from the distance of the primary intervals (internally and externally) by multiplying adjacent primary intervals by the Fibonacci ratios $0.618,1.618,0.786$ (square root of 0.618 ), and 1.272 (square root of 1.618). The method halts after two iterations for simplicity as a global con- 
straint [5]. The usefulness of the square root ratios (i.e., 0.786 and 1.272) could be called into question since the main idea was to come up with a general golden ratio based parameterization of the primary intervals. Although, the square root of the golden ratio (1.27) has astronomical significance in the ratio of the diameters of earth and moon and biological significance in the median insect growth ratio [29].

The HM presents a novel general closed-form, continuous forward model of the primary intervals. This leads to phase-constant temporal patterns. Contrary to the GSM, no external constraints within the model are required, except the standard assumption of halting temporal patterns at the average human life expectancy. For simplicity, the fewer assumptions imposed by the model, the better, and so the HM may be considered simpler than the GSM. Interestingly, the Fibonacci sequence itself is a sinusoidal waveform with the ratios of adjacent terms progressing in an alternating bigger $(+)$ and smaller $(-)$ pattern, and the 24 repeating period of the Fibonacci numbers is approximately sinusoidal [9]. Harmonics, which causes resonance in the sinusoidal waveform, is usually considered as an integer multiple of the fundamental frequency. This form of harmonics is called "integer harmonics" or simply "harmonics" since it is common. However, the present research deals with a slightly more complicated form of harmonics called "non-integer harmonics." Non-integer harmonics are harmonics that are a non-integer multiple of the fundamental frequency. Specifically, the harmonics frequencies observed in this research are powers of Phi fractions of the fundamental frequency.

\subsection{Generality}

The models carry several advantages for generality, including drawing from scientific disciplines ranging from mathematics and physics to biology and psychology. There are multiple sources of the widespread appearance of the Fibonacci numbers and ratio in the natural universe. For example, an overall optimum healthy heart function occurs when there is convergence of the Fibonacci numbers and Phi relationship between the waves on the electrocardiogram [30]. It is dramatically seen in the shape of spiral galaxies. Fibonacci resonance can explain why the orbital distances of planets and hence by Kepler's laws their orbital periods are found at golden ratio distances from the Sun [31], and why electrons abide by quantum levels [18]. Findings based on the study of data from cosmic background radiation reveal that the universe is shaped like a dodecahedron, a geometric shape based on pentagons, which are based on Phi [32]. Fibonacci resonance is also found in Mandelbrot's fractal [13], suggesting it to be the basic dynamic fractal of the universe.

Both models have several clinical implications. One possible avenue for psychotherapy practice could be to assist therapists in their work with adults coping with life transitions. Insofar as Fibonacci numbers predict attractors, fractals, and chaos [11] [13], it can reduce the risks of counsellors missing crucial op- 
portunities or focusing on irrelevant issues, such as by charting clients' adaptive landscapes, which allows the counsellor to attend to system self-organization and emergent dynamics. Also, it allows both subjective and objective information to be portrayed; permits an improved understanding about the self by relating past experiences; and allows clients to experience improved understanding of synchronicity experiences that occur during phase transitions. This dynamical systems approach allows compatibility with a wide range of counseling approaches, styles, or theories. For example, this kind of cognitive reflection may be combined with mindfulness-based practices [33].

The models proposed in this paper apply best to synchronicity broadly construed. They are less applicable to more ordinary spiritual experiences (because of the lack of nonlinear dynamics, and because of their wide variability). As William James noted [34], spiritual experiences are diverse. Many spiritual experiences (e.g., awe and joy, watching a sunset) do not infringe "everyday" causal principles, and may not emphasize the sense of dramatic change. In contrast, the basis of synchronicity is altered perceived causality and meaningfulness that facilitates a feeling of sudden change. Often there is a sense in which the synchronicity occurs as part of a sequence of events with a central theme. Viewed in this light, synchronicity can be seen as a configuration in neural networks in which a sequence of events pushes the system into a new emergent state leading to a phase transition. Therefore, the models are a preliminary comprehensive model of synchronicity based on dynamic systems principles.

\section{Discussion and Conclusion}

This article had two purposes. First, it sought to elaborate a harmonic model of synchronicity based on the Fibonacci Life Chart Method (FLCM). Second, it sought to compare the golden section model (GSM) and harmonic model (HM). The development of the HM was treated as a separate task from the model comparison, and some explanations and predictions were developed that can be tested in future data.

The analysis of synchronicity as Fibonacci time patterns appears capable of supporting a broad range of testable predictions. It is also a useful link between mathematics and synchronicity. That is, the author followed Jung [6] in suggesting that Fibonacci numbers can furnish a plausible explanation for synchronicity, but the theory itself is essentially dynamical in the sense that it specifies how change will be meaningfully interlinked and organized. Like modern physics research itself, the FLCM draws on dynamical systems theory and its understanding of change dynamics, self-organization, emergence, and fractal dynamics. Thus, the present theory is dynamical, because it proposes that synchronicity will operate based on Fibonacci numbers controlling the interplay of genes and brain development, which change coherence of brain waves, which cycle around again to influence brain development.

The second part of the article compared the two models, and they were gener- 
ally consistent with many aspects of dynamical systems theory. Both models have several essential features such as Fibonacci time patterns and nonlinear dynamics consistent with the view that synchronicity is often a sudden change in the lives of many people. Thus, in dynamical systems theory complex systems are prone to exhibit extreme sensitivity to perturbations, so that nonlinear causality among interacting components allows small differences to produce large effects over time. This classic feature of dynamic systems is known as the "butterfly effect": the butterfly's wings can influence air currents that, over many iterations, result in a thunderstorm. But if self-organizing cognition-emotion interactions are so sensitive, how do they attain the coherence and resiliency that characterize personality? And how do they attain consistency within, and sometimes across, individuals, as highlighted by conventional norms in society and culture? These questions lead to a reconsideration of the sources of orderliness in development and personality (see Table 1). These seemingly contradictory observations can be reconciled through an understanding of Fibonacci time patterns, which show that attractor dynamics are insensitive to perturbation, whereas the dynamic trajectory of the system is extremely sensitive to conditions near fractal boundaries.

On any given state in the phase space diagram, human development gravitates toward attractor states that are codetermined by genetic, cultural, and experiential histories. Biological and cultural constraints influence the way cognitive elements cohere together and the way cognitions and emotions reciprocally activate each other, but these constraints are continuously modified by the emergent structure of biological unfolding of events involved in a person changing gradually from a simple to a more complex level. Both universal and idiosyncratic constraints thus guide human development, allowing for normative themes and individual variations in cognition-emotion interactions.

In general, the data suggest that FLCM ages have strong predictive value as points of discontinuity, whereas non-FLCM ages command a significant source of continuity. Most of the findings pertain to biological and psychological changes, and it seems reasonable to conclude that Fibonacci time patterns predict the evolution of trajectories in phase space [11]. There is less evidence to indicate that Fibonacci time patterns continue to be relevant to synchronicity experiences, though some findings (such as involving religious and spiritual experiences) do suggest that the dynamical effects of age 18 are a predictor of spiritual experience [14]. FLCM ages specify exclusive discontinuity and change, as the person is removed from points of stability in dynamical phase space, and the psychological processes of that phase space are more relevant to nonlinear and fractal dynamics.

Synchronicity is a phenomenon that disrupts conventional notions of temporal ordering, and so it would be surprising if mathematics and dynamical principles were absent. I do not seek to replace all other theories of spiritual experience with this mathematical one, and I have noted that the mathematical anal- 
ysis does not apply equally well to all spiritual experiences. I propose only that the mathematical analysis deserves to be included as one important dimension of synchronicity experience and one useful approach for understanding the nonlinear dynamics of synchronicity, especially the large-scale, personality transforming experiences of synchronicity. Considering how well the FLCM model fits the developmental data, I think it appropriate that researchers interested in the mathematical aspects of synchronicity can begin conducting more prospective and explicit tests of it.

\section{References}

[1] Lloyd, S. (2010) The Computational Universe. In: Davies, P. and Gregersen, N.H., Eds., Information and the Nature of Reality: From Physics to Metaphysics, Cambridge University Press, Cambridge, 92-103. https://doi.org/10.1017/CBO9780511778759.005

[2] Tegmark, M. (2014) Our Mathematical Universe: My Quest for the Ultimate Nature of Reality. Vintage Books, New York.

[3] Atmanspacher, H. (2012) Dual-Aspect Monism à la Pauli and Jung. Journal of Consciousness Studies, 19, 96-120.

[4] Johansen, M.K. and Osman, M. (2015) Coincidences: A Fundamental Consequence of Rational Cognition. New Ideas in Psychology, 39, 34-44. https://doi.org/10.1016/j.newideapsych.2015.07.001

[5] Sacco, R.G. (2016) The Fibonacci Life-Chart Method (FLCM) as a Foundation for Carl Jung's Theory of Synchronicity. Journal of Analytical Psychology, 61, 203-222. https://doi.org/10.1111/1468-5922.12204

[6] Jung, C.G. (1976) Letters of C. G. Jung: Vol. 2, 1951-1961. Routledge, London.

[7] Vallacher, R.R., Read, S.J. and Nowak, A. (2017) Computational Social Psychology. Routledge, New York.

[8] Thelen, E. and Smith, L.B. (1994) A Dynamic Systems Approach to the Development of Perception and Action. MIT Press, Cambridge.

[9] Sacco, R.G. (2013) Re-Envisaging the Eight Developmental Stages of Erik Erikson: The Fibonacci Life-Chart Method (FLCM). Journal of Educational and Developmental Psychology, 3, 140-146. https://doi.org/10.5539/jedp.v3n1p140

[10] Lagarkova, M.A., Eremeev, A.V., Svetlakov, A.V., Rubtsov, N.B. and Kiselev, S.L. (2010) Human Embryonic Stem Cell Lines Isolation, Cultivation, and Characterization. In Vitro Cellular and Developmental Biology-Animal, 46, 284-293. https://doi.org/10.1007/s11626-010-9282-6

[11] Linage, G., Montoya, F., Sarmiento, A., Showalter, K. and Parmananda, P. (2006) Fibonacci Order in the Period-Doubling Cascade to Chaos. Physics Letters, 359, 638-639. https://doi.org/10.1016/j.physleta.2006.07.036

[12] Bak, P. (1996) How Nature Works: The Science of Self-Organized Criticality. Springer, New York. https://doi.org/10.1007/978-1-4757-5426-1

[13] Devaney, R.L. (1999) The Mandelbrot Set, the Farey Tree, and the Fibonacci Sequence. The American Mathematical Monthly, 106, 289-302. https://doi.org/10.1080/00029890.1999.12005046

[14] Sacco, R.G. (2017) The Fibonacci Life Chart Method as a Predictor of Spiritual Experience. Journal of Educational and Developmental Psychology, 7, 1-13. 
https://doi.org/10.5539/jedp.v7n2p1

[15] Katznelson, Y. (2004) An Introduction to Harmonic Analysis. 3rd Edition, Cambridge University Press, Cambridge. https://doi.org/10.1017/CBO9781139165372

[16] Centers for Disease Control and Prevention (2017) Mortality in the United States, 2016. https://www.cdc.gov/nchs/data/databriefs/db293.pdf

[17] Aoyama, T., Hayakawa, M., Kinoshita, T. and Nio, M. (2012) Tenth-Order QED Contribution to the Electron G-2 and an Improved Value of the Fine Structure Constant. Physical Review Letters, 109, Article ID: 111807. https://doi.org/10.1103/PhysRevLett.109.111807

[18] Coldea, R., Tennant, D.A., Wheeler, E.M., Wawrzynska, E., Prabhakaran, D., Telling, M., Kiefer, K., et al. (2010) Quantum Criticality in an Ising Chain: Experimental Evidence for Emergent $\mathrm{E}_{8}$ Symmetry. Science, 327, 177-180. https://doi.org/10.1126/science.1180085

[19] Mitchell, E.D. and Staretz, R. (2011) The Quantum Hologram and the Nature of Consciousness. Journal of Cosmology, 14.

[20] Maxwell, N. (2003) The Comprehensibility of the Universe: A New Conception of Science. Oxford University Press, Oxford. https://doi.org/10.1093/acprof:oso/9780199261550.001.0001

[21] Cambray, J. (2009) Synchronicity: Nature and Psyche in an Interconnected Universe. Texas A \& M University Press, College Station.

[22] Hogenson, G.B. (2005) The Self, the Symbolic and Synchronicity: Virtual Realities and the Emergence of the Psyche. Journal of Analytical Psychology, 50, 271-284. https://doi.org/10.1111/j.0021-8774.2005.00531.x

[23] Mansfield, V. (1995) Synchronicity, Science, and Soulmaking: Understanding Jungian Synchronicity through Physics, Buddhism, and Philosophy. Open Court, Chicago.

[24] Jung, C.G. (1952) Synchronicity: An Acausal Connecting Principle. CW 8.

[25] Atasoy, S., Deco, G., Kringelbach, M.L. and Pearson, J. (2017) Harmonic Brain Modes: A Unifying Framework for Linking Space and Time in Brain Dynamics. The Neuroscientist, 24, 277-293. https://doi.org/10.1101/162040

[26] Roopun, A.K., Kramer, M.A., Carracedo, L.M., Kaiser, M., Davies, C.H., Traub, R. D., et al. (2008) Temporal Interactions between Cortical Rhythms. Frontiers in Neuroscience, 2, 145-154. https://doi.org/10.3389/neuro.01.034.2008

[27] Hitchcock, C. (2018) Probabilistic Causation. Stanford Encyclopedia of Philosophy. https://plato.stanford.edu/entries/causation-probabilistic

[28] Costin, G., Dzara, K. and Resch, D. (2011) Synchronicity: Coincidence Detection and Meaningful Life Events. Psychiatric Annals, 41, 572-575.

https://doi.org/10.3928/00485713-20111104-04

[29] Cole, B.J. (1980) Growth Ratios in Holometabolous and Hemimetabolous Insects. Annals of the Entomological Society of America, 73, 489-491. https://doi.org/10.1093/aesa/73.4.489

[30] Henein, M.Y., Collaborators, G.R., Zhao, Y., Nicoll, R., Sun, L., Khir, A.W., Lindqvist, P., et al. (2011) The Human Heart: Application of the Golden Ratio and Angle. International Journal of Cardiology, 150, 239-242. https://doi.org/10.1016/j.ijcard.2011.05.094

[31] Tattersall, R. (2013) The Hum: Log-Normal Distribution and Planetary-Solar Resonance. Pattern Recognition in Physics, 1, 185-198.

https://doi.org/10.5194/prp-1-185-2013 
[32] Luminet, J.P. (2016) The Status of Cosmic Topology After Planck Data. Universe, 2, 1. https://doi.org/10.3390/universe2010001

[33] Gu, J., Strauss, C., Bond, R. and Cavanagh, K. (2015) How Do Mindfulness-Based Cognitive Therapy and Mindfulness-Based Stress Reduction Improve Mental Health and Wellbeing? A Systematic Review and Meta-Analysis of Mediation Studies. Clinical Psychology Review, 37, 1-12. https://doi.org/10.1016/j.cpr.2015.01.006

[34] James, W. (1902/1985) The Varieties of Religious Experience. Harvard University Press, Cambridge. 